\title{
Considerações sobre a prevenção do câncer de mama feminino
}

\author{
Considerations on the prevention of female breast cancer
}

\author{
Luiz Claudio Thuler'
}

\section{Resumo}

O controle de câncer de mama deve priorizar a prevenção e a detecção precoce. A prevenção não deve focalizar apenas os fatores de risco associados ao câncer de mama mas também os fatores de proteção. Alguns fatores que aumentam o risco de desenvolver câncer de mama, como obesidade na pós-menopausa, exposição à radiação ionizante em altas doses, exposição a pesticidas/organoclorados e tabagismo são passíveis de intervenção; outros fatores como sexo feminino, avanço da idade, menarca precoce, menopausa tardia, primeira gestação tardia, história de câncer de ovário ou de mama ou história de doença mamária benigna, alta densidade mamária, mutações genéticas (BRCA1 e BRCA2) e história familiar de câncer de mama não podem ser modificados. A maioria destes fatores encontra-se associada com um moderado aumento no risco (cerca de 2 ou 3 vezes), o que sugere que múltiplos fatores contribuem para a gênese da doença e que podem existir fatores ainda desconhecidos. Por outro lado, há duas estratégias principais para a detecção precoce do câncer de mama: a educação para promover o diagnóstico precoce e o rastreamento. As evidências obtidas de ensaios clínicos sugerem uma diminuição de $25 \%$ da mortalidade por câncer de mama com o rastreamento mamográfico de rotina. Além disso, há evidências indiretas de que o rastreamento por exame clínico das mamas reduza o número de mortes por este câncer. Palavras-chave: neoplasias mamárias; prevenção e controle; fatores de risco; detecção precoce.

\section{Abstract}

Breast cancer control should place emphasis on preventing cancers or detecting cases in early stages. Prevention not only focuses on the risks associated with breast cancer but also on protective factors. Some factors that increase breast cancer risk, such as postmenopausal obesity, high-dose exposure to ionizing radiation, exposure to pesticides/organochlorines and tobacco use, are modifiable; other risk factors, such as female gender, age, early menarche, late menopause, late first full-term pregnancy, previous breast and ovarian cancer, benign breast disease, high breast density, genetic mutations (BRCA1 and BRCA2), or family history of breast cancer, cannot be modified. Most of these risk factors are associated with only a moderate increase in risk (two- or three fold), suggesting that multiple factors may play a role in each woman's disease and that unrecognized factors may exist. On the other hand, there are two principal components for early detection: education to promote early diagnosis, and screening. The evidence available to date, from population-based controlled trials examining the effectiveness of routine screening with mammography suggests a $25 \%$ reduction in breast cancer mortality. In addition, there is only indirect evidence that screening by clinical breast examination will reduce the number of breast cancer deaths. Key words: breast neoplasms; prevention e control; risk factors; early detection.

${ }^{1}$ Médico epidemiologista do Insituto Nacional de Câncer - Ministério da Saúde. 


\section{INTRODUÇÃO}

Estratégias de prevenção primária, secundária e terciária têm sido utilizadas com o objetivo de prevenir enfermidades, diagnosticá-las e tratá-las precocemente e minimizar seus efeitos na população, assegurando, a cada indivíduo, um padrão de vida adequado à manutenção da sua saúde ${ }^{1}$. Estes conceitos clássicos da Saúde Pública levam em conta a história natural das doenças, ao considerar que, após a exposição aos fatores de risco, inicia-se o processo patológico, surgem sinais e sintomas e há progressão para cura, incapacidade ou morte. Cada um desses eventos marca o início de uma nova etapa, conforme apresentado na figura 1 . Neste contexto, as diferentes fases de progressão da doença incluem um período de indução (fase de suscetibilidade), seguido de um estágio pré-clínico, de latência ou assintomático (equivalente ao período de incubação nas doenças infecciosas) e uma fase clínica ou sintomática.

Figura 1. Estratégias de prevenção conforme o estágio de progressão da doença.

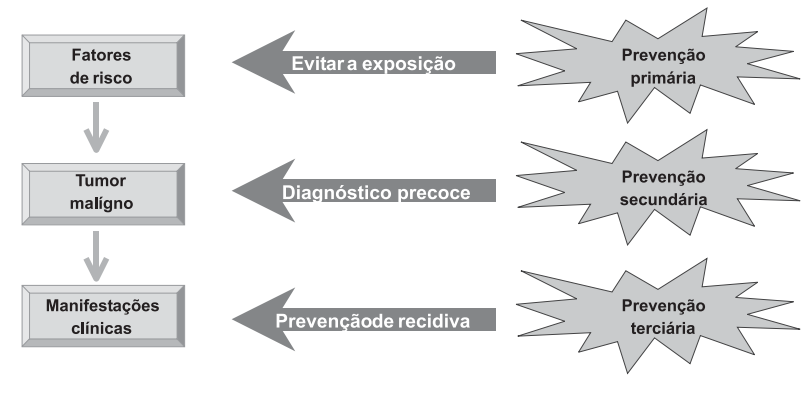

Desta forma, a principal distinção entre prevenção primária, secundária e terciária repousa no período de progressão da doença sobre o qual se deseja intervir: antes da instalação do processo patológico (prevenção primária), depois que a doença se iniciou e ainda não há sintomas (prevenção secundária) ou quando já há sintomas (prevenção terciária).

\section{PreVEnção PRIMÁrIa}

As açōes de prevenção primária objetivam diminuir a incidência de uma doença numa população, reduzindo o risco de surgimento de casos novos, ao prevenir a exposição aos fatores que levam ao seu desenvolvimento, interromper seus efeitos ou alterar as respostas do hospedeiro a essa exposição, impedindo que ocorra seu início biológico. Alguns exemplos dessas estratégias podem ser encontrados em programas que promovem práticas sexuais seguras, ajudam pessoas a deixar de fumar e realizam vacinação em massa, respectivamente.

Partindo-se da lógica de que características que aumentam a probabilidade de desenvolver uma condição mórbida representem fatores de risco ${ }^{2}$, seu controle, por intermédio de intervenções clínico-epidemiológicas, permitirá evitar ou minimizar os agravos à saúde. A medida do risco geralmente é feita por meio do cálculo do risco relativo ou da razão dos riscos. Um risco relativo igual a um $(\mathrm{RR}=1)$ significa que a probabilidade individual de desenvolver a doença é a mesma que aquela observada em pessoas que não estão expostas ao fator em questão. Um risco relativo maior que um $(R R>1)$ significa uma probabilidade aumentada, enquanto os valores menores que um $(R R<1)$ indicam proteção ou ausência de risco. Além disso, quando o intervalo com 95\% de confiança inclui a unidade, a associação entre o fator de risco ou proteção e a doença não é estatisticamente significativa. Alguns autores mais conservadores sugerem considerar somente riscos maiores que dois ou três, afirmando que valores menores quase sempre estão sujeitos a confundimento ou viés ${ }^{3}$.

No que diz respeito à identificação dos fatores de risco, embora não haja uniformidade na sua definição e método de mensuração e apesar das contradiçôes observadas entre os diferentes estudos, o sexo, a idade, a história familiar e a história pregressa de câncer de mama, a história reprodutiva e a suscetibilidade genética têm sido apontados como associados a um risco aumentado de desenvolver câncer de mama (tabela 1).

O simples fato de pertencer ao sexo feminino constitui-se no fator de risco mais importante. Embora homens possam apresentar este tipo de câncer, a doença é pelo menos 100 a 150 vezes mais freqüente entre as mulheres ${ }^{4,5}$. Isto se deve à maior quantidade de tecido mamário encontrado nas mulheres e à sua exposição ao estrogênio endógeno.

No sexo feminino, a maior contribuição para a gênese do câncer de mama vem da idade, sendo o envelhecimento o único fator de risco conhecido em muitos casos ${ }^{6}$. No Brasil, para o período compreendido entre 1996 e 2000, dados de incidência dos Registros de Câncer de Base Populacional de Goiânia ${ }^{7}$, São Paulo ${ }^{8}$ e Manaus ${ }^{9}$ indicam que 60 a $70 \%$ dos casos novos ocorreram na faixa etária compreendida entre os $40 \mathrm{e}$ 69 anos de idade. No que diz respeito à mortalidade por câncer de mama, dados disponíveis para o período de 1996 a 2000 mostram que $91 \%$ das mortes foram registradas em mulheres com mais de 40 anos de idade, conforme pode ser observado na tabela 2 . 
Tabela 1. Principais fatores associados ao desenvolvimento do câncer de mama. (Adaptado de FLETCHER5; HEALTH CANADA6; ROBLES \& GALANIS10; MCPHERSON, STEEL \& DIXON11)

\begin{tabular}{|c|c|c|c|c|}
\hline \multirow[b]{2}{*}{ FATOR } & \multicolumn{4}{|c|}{ RISCO RELATIVO* } \\
\hline & $\begin{array}{l}\text { HEALTH } \\
\text { CANADA }\end{array}$ & FLETCHER & $\begin{array}{c}\text { ROBLES \& } \\
\text { GALANIS }\end{array}$ & $\begin{array}{l}\text { MCPHERSON, } \\
\text { STEEL \& DIXON }\end{array}$ \\
\hline \multicolumn{5}{|l|}{ Sexo } \\
\hline Feminino $\mathrm{x}$ masculino & --- & 150,0 & --- & --- \\
\hline \multicolumn{5}{|l|}{ Idade } \\
\hline$>50 x<50$ anos & 4,0 & --. & --- & --- \\
\hline $70-74 \times 30-34$ anos & --- & 17,0 & --- & --- \\
\hline Mais velhas & --- & --- & --- & $>10,0$ \\
\hline \multicolumn{5}{|l|}{ Idade da menarca: } \\
\hline $11 \times 15$ anos & $1,1-2,0$ & $--\cdot$ & --- & --- \\
\hline$<12 x>14$ anos & --- & 1,5 & --- & --- \\
\hline$>14$ anos $x<12$ anos & --- &.-- & 0,77 & --- \\
\hline Paridade: & --- & $--\cdot$ & --- & --- \\
\hline Nuliparidade $x$ paridade & $1,1-2,0$ & --- & $1,2-1,7$ & --- \\
\hline Nuliparidade $\times 5$ gestações & --- & --- & 2,0 & --- \\
\hline \multicolumn{5}{|l|}{ Idade do primeiro parto: } \\
\hline$>40$ anos & --- & --- & 3,0 & --- \\
\hline$>30$ anos $x<20$ anos & $1,1-3,0$ & $1,9-3,5$ & --- & --- \\
\hline$>35$ anos $x<20$ anos & --- & --- & 1,4 & --- \\
\hline$\geq 35$ anos $x$ nulípara & --- & --- & 1,2 & --- \\
\hline \multicolumn{5}{|l|}{ Idade da Menopausa: } \\
\hline $55 \times 45$ anos & $1,1-2,0$ & $--\cdot$ & --- & --- \\
\hline$>55 x<45$ anos & --- & 2,0 & 2,0 & --- \\
\hline$>54$ anos & --- & --- & --- & 2,0 \\
\hline \multicolumn{5}{|l|}{ Idade da ooforectomia: } \\
\hline$<40$ anos & --- & --- & 0,5 & --- \\
\hline$>40$ anos & $1,1-3,0$ & --- & --- & --- \\
\hline$>35$ anos $x<35$ anos & --- & 3,0 & --- & --- \\
\hline \multicolumn{5}{|l|}{ Raça / etnia: } \\
\hline $\begin{array}{l}<45 \text { anos de idade: branca } \times \text { hispânica e } \\
\text { asiática }\end{array}$ & $1,1-2,0$ & $--\cdot$ & --- & --- \\
\hline$<40$ anos de idade: negra x hispânica e asiática & $1,1-2,0$ & --- & --- & --- \\
\hline \multicolumn{5}{|l|}{ Classe sócio-econômica: } \\
\hline Alta $x$ baixa & $1,1-2,0$ &.-- & --- & 2,0 \\
\hline $2 / 3$ maiores $\times 1 / 3$ menor & --- & --- & $1,7(1,2-2,4)$ & --- \\
\hline \multicolumn{5}{|l|}{ Religião: } \\
\hline Judia $\times$ Adventista de $7^{\circ}$ dia ou mormon & $1,1-2,0$ & --- & --- & --- \\
\hline \multicolumn{5}{|l|}{ País de nascimento: } \\
\hline $\begin{array}{l}\text { Ásia e África X América do Norte e Nordeste da } \\
\text { Europa }\end{array}$ & $<0,3$ & --- & --- & --- \\
\hline \multicolumn{5}{|l|}{ Local de residência: } \\
\hline Urbano x rural & $1,1-2,0$ & $--\cdot$ & --- & --- \\
\hline País subdesenvolvido & --- & --- & 0,2 & --- \\
\hline \multicolumn{5}{|l|}{ Estado Civil: } \\
\hline Nunca casou x casada pelo menos uma vez & $1,1-2,0$ & $--\cdot$ & --- & --- \\
\hline \multicolumn{5}{|l|}{ História pregressa de : } \\
\hline Câncer de mama contralateral & --- & --- & $2,0-5,0$ & $>4,0$ \\
\hline Câncer de ovário & $1,1-2,0$ & --- & $1,4-4,0$ & --- \\
\hline Câncer de endométrio & $1,1-2,0$ & --- & $1,2-2,0$ & --- \\
\hline \multicolumn{5}{|l|}{ Mutação genética: } \\
\hline BRCA 1 & \multirow{2}{*}{$>4,0$} & --- & $27(8,0-89,0)$ &.-- \\
\hline BRCA 2 & & --- & $2(0,05-12,0)$ & --- \\
\hline
\end{tabular}




\begin{tabular}{|c|c|c|c|c|}
\hline \multirow[b]{2}{*}{ FATOR } & \multicolumn{4}{|c|}{ RISCO RELATIVO* } \\
\hline & $\begin{array}{l}\text { HEALTH } \\
\text { CANADA }\end{array}$ & FLETCHER & $\begin{array}{c}\text { ROBLES \& } \\
\text { GALANIS }\end{array}$ & $\begin{array}{l}\text { McPHERSON, } \\
\text { STEEL \& DIXON }\end{array}$ \\
\hline \multicolumn{5}{|l|}{ História de doença mamária benigna: } \\
\hline Ausência de doença proliferativa & --- & $\ldots$ & $0,9-1,6$ & -.. \\
\hline Doença proliferativa sem atipia & $2,0-4,0$ & -- & $1,6-2,2$ & $\ldots$ \\
\hline Hiperplasia atípica & $>4,0$ & 1,8 & $2,5-5,3$ & $4,0-5,0$ \\
\hline Células epiteliais hiperplasicas, sem atípia & $2,0-4,0$ & -.. & -.- & ... \\
\hline Fibroadenoma & --- & -- & $0,47-1,7$ & -.. \\
\hline Doença cística de Gross & --- & --- & $1,8-4,4$ & -.. \\
\hline Carcinoma "in situ" & $>4,0$ & --- & $5,9-12,0$ & -.. \\
\hline \multicolumn{5}{|l|}{ Densidade mamária: } \\
\hline Níveis mais elevados $\mathrm{x}$ níveis mais baixos & --- & --- & $4,04-6,05$ & -.- \\
\hline$>75 \%$ / tecido gorduroso & $3,0-4,0$ & -- & --- & -.- \\
\hline $65 \%$ de densidade mamária & --- & --- & 4,3 & -.. \\
\hline \multicolumn{5}{|l|}{ História familiar de câncer de mama: } \\
\hline Um familiar & --. & 2,6 & -.- & ... \\
\hline Um familiar de primeiro grau & $2,0-4,0$ & --- & $1,8-2,6$ & $\geq 2,0$ \\
\hline Dois familiares de primeiro grau & --- & --- & $2,5(1,5-4,2)$ & -.. \\
\hline Familiar de segundo grau & --- & --- & $\begin{array}{c}1,82(1,39- \\
2,24)\end{array}$ & $-\cdots$ \\
\hline Familiar de terceiro grau & --- & --- & $\begin{array}{c}1,35(1,07- \\
1,64)\end{array}$ & $-\cdots$ \\
\hline \multicolumn{5}{|l|}{ Uso de contraceptivo oral: } \\
\hline Uso corrente x nunca & 1,5 & $1,07-1,2$ & $\begin{array}{c}1,24(1,15- \\
1,33)\end{array}$ & 1,24 \\
\hline Uso corrente $x>10$ anos sem usar & --- & --- & --- & $\begin{array}{c}1,24(0,96- \\
1,05)\end{array}$ \\
\hline Início antes dos 20 anos $x$ tardio & --- & --- & $\begin{array}{c}1,22(1,18- \\
1,26)\end{array}$ & $-\cdot-$ \\
\hline \multicolumn{5}{|l|}{ Estrogenioterapia: } \\
\hline Uso freqüente $\mathrm{x}$ nunca & -- & $1,2-1,4$ & --- & -.- \\
\hline \multicolumn{5}{|l|}{ Dietilbestrol: } \\
\hline Uso durante a gravidez x não uso & --- & -- & 1,5 & 2,0 \\
\hline \multicolumn{5}{|l|}{ Uso de depoprovera: } \\
\hline Alguma vez $\mathrm{x}$ nunca & --- & --- & $1,1(0,97-2,2)$ & -.- \\
\hline Uso freqüente & --- & --- & $1,5(1,0-2,2)$ & -.- \\
\hline Primeiro uso há $<5$ anos & --- & --- & $2,0(1,5-2,8)$ &.-- \\
\hline \multicolumn{5}{|l|}{ Terapia de reposição hormonal: } \\
\hline Uso corrente há $<5$ anos & --- & -- & $1,07(0,72-1,12)$ & -.- \\
\hline Uso corrente há $>5$ anos & --- & --- & $\begin{array}{c}1,35(1,21- \\
1,49)\end{array}$ & $--\cdot$ \\
\hline Uso $\geq 10$ anos & -.- & -.- & --- & 1,35 \\
\hline \multicolumn{5}{|l|}{ Tamoxifeno: } \\
\hline Uso x não uso & -- & -- & 0,55 & -.. \\
\hline \multicolumn{5}{|l|}{ Gorduras alimentares: } \\
\hline Aumento de $100 \mathrm{~g} /$ dia na gordura alimentar & --- & --- & 1,35 & -.. \\
\hline Maior $\mathrm{x}$ menor categoria de ingesta de gordura & -- & -- & 1,03 & 1,5 \\
\hline \multicolumn{5}{|l|}{ Fibras alimentares: } \\
\hline Aumento de $20 \mathrm{~g} /$ dia na ingestão de fibras & --- & --- & 0,85 & -.. \\
\hline Alto $x$ baixo consumo de fibras & --- & --- & $\begin{array}{c}1,02(0,85- \\
1,23)\end{array}$ & $--\cdot$ \\
\hline \multicolumn{5}{|l|}{ Obesidade: } \\
\hline Na pré-menopausa & --- & --- & $0,6-1,3$ & 0,7 \\
\hline Na pós-menopausa & -.- & 1,6 & $1,0-2,7$ & 2,0 \\
\hline
\end{tabular}




\begin{tabular}{|c|c|c|c|c|}
\hline \multirow[b]{2}{*}{ FATOR } & \multicolumn{4}{|c|}{ RISCO RELATIVO* } \\
\hline & $\begin{array}{l}\text { HEALTH } \\
\text { CANADA }\end{array}$ & FLETCHER & $\begin{array}{c}\text { ROBLES \& } \\
\text { GALANIS }\end{array}$ & $\begin{array}{l}\text { MCPHERSON, } \\
\text { STEEL \& DIXON }\end{array}$ \\
\hline \multicolumn{5}{|l|}{ Altura: } \\
\hline$>1,70 \mathrm{~m}$ & --- & --- & $0,9-2,1$ & --- \\
\hline \multicolumn{5}{|l|}{ Ingesta de álcool: } \\
\hline 10g/dia ( 1 drink) x não uso & --- & --- & $\begin{array}{c}1,09(1,04- \\
1,13)\end{array}$ & --- \\
\hline$>24 \mathrm{~g} /$ dia (>2 drinks) x não uso & --- & --- & $1,04(1,0-1,8)$ & --- \\
\hline$>40 \mathrm{~g} /$ dia (>3 drinks) $\times$ não uso & --- & --- & 1,69 & --- \\
\hline Consumo excessivo & --- & --- & --- & 1,3 \\
\hline \multicolumn{5}{|l|}{ Tabagismo: } \\
\hline$\geq 20$ cigarros/dia & --- & --- & $0,57-2,9$ & --- \\
\hline \multicolumn{5}{|l|}{ Radiação iônica do tórax: } \\
\hline Exposição x não exposição & $2,0-4,0$ & --- & $1,4-2,2$ & 3,0 \\
\hline \multicolumn{5}{|l|}{ Pesticidas / organoclorados: } \\
\hline Exposição x não exposição & --- & --- & $0,66-4,08$ & --- \\
\hline
\end{tabular}

*Nota: Números apresentados isoladamente referem-se à estimativa pontual do risco; Números separados por hífen representam a variação da estimativa de risco encontrada entre os diferentes estudos; Números entre parênteses separados por hífen referem-se ao intervalo com $95 \%$ de confiança.

Outro importante fator para o desenvolvimento do câncer de mama é a predisposição genética. Observa-se um risco aumentado em mulheres com casos da doença em familiares próximos (mãe, irmã ou filha). Este risco é especialmente elevado quando o familiar tem câncer antes dos 50 anos de idade e em ambas as mamas. Apesar de sua importância, de acordo com a Associação Americana de Câncer, a maioria das mulheres com câncer de mama (aproximadamente $80 \%$ ) não tem história familiar da doença e somente cerca de 5 a $10 \%$ dos casos de câncer de mama podem ser atribuídos a mutações genéticas ${ }^{4}$. Muitas vezes as mutações ocorrem nos genes BRCA1 e BRCA2, implicando um elevado risco de desenvolver a doença, sendo metade dos casos antes dos 50 anos de idade. Entretanto, observa-se que somente uma em cada 1000 mulheres apresenta este padrão de mutação ${ }^{5,12}$.

Tabela 2. Distribuição percentual dos casos e óbitos por câncer de mama, no Brasil, no período compreendido entre 1996 e 2000.

\begin{tabular}{|c|c|c|c|c|}
\hline \multirow{2}{*}{$\begin{array}{c}\text { Faixa Etária } \\
\text { (em anos) }\end{array}$} & \multicolumn{3}{|c|}{ casos novos* (\%) } & \multirow{2}{*}{ óbitos* (\%) } \\
\cline { 1 - 5 } Até 29 & 1,7 & 1,4 & 3,1 & 0,9 \\
\hline 30 a 39 & 14,5 & 9,1 & 10,1 & 7,8 \\
\hline 40 a 49 & 34,3 & 21,0 & 31,0 & 19,8 \\
\hline 50 a 59 & 20,9 & 22,2 & 18,6 & 23,9 \\
\hline 60 a 69 & 9,9 & 18,0 & 20,2 & 21,1 \\
\hline 70 e mais & 18,6 & 20,0 & 15,5 & 26,3 \\
\hline Idade ignorada & 0 & 8,4 & 1,6 & 0,1 \\
\hline Total & 100,0 & 100,0 & 100,0 & 100,0 \\
\hline
\end{tabular}

Fontes: *Registros de Câncer de Base Populacional de Goiânia $(1997)^{7}$, São Paulo $(1998)^{8}$ e Manaus $(1999)^{9}$; ${ }^{* *}$ Sistema de Informação de Mortalidade (média 1996 a 2000) ${ }^{13}$.
Sabe-se que o estrogênio tem um importante papel no câncer de mama ao induzir o crescimento das células do tecido mamário, o que aumenta o potencial de alterações genéticas e, conseqüentemente, o desenvolvimento do câncer. Por isso, qualquer fator que leve a um aumento no estrogênio poderá levar também a um aumento no risco de adoecer por câncer de mama ${ }^{10}$. Mulheres com história de menarca precoce, primeiro filho em idade avançada, obesidade na pósmenopausa, câncer de ovário, densidade mamária elevada, doença mamária benigna, exposição ao tabaco, a radiaçóes ionizantes e pesticidas/organoclorados apresentam aumento no risco de desenvolver câncer de mama. Além disso, mulheres que tiveram câncer em uma das mamas apresentam um elevado risco de desenvolver a doença na mama contra-lateral ${ }^{12}$.

Especula-se ainda que os seguintes fatores estejam associados ao câncer de mama, apesar de faltarem evidências científicas até o presente momento: abortamento, aumento do volume das mamas, baixa ingesta de fitoestrogênios e uso de drogas antiinflamatórias ${ }^{4}$. Por outro lado, os seguintes fatores parecem não estar associados ao câncer de mama: exposição a campos magnéticos, trauma mamário, uso de antitranspirantes e de implantes de próteses mamárias de silicone $e^{4,14}$.

Por fim, o conhecimento científico acumulado até o presente indica que menos de $50 \%$ dos casos de câncer de mama podem ser explicados pelos principais fatores de risco descritos ${ }^{9,15}$. Infelizmente há pouco que se possa fazer para evitar a exposição à maioria destes fatores. Entretanto, não fumar, manter o peso adequado após a 
menopausa e evitar a exposição à radiação iônica e aos pesticidas/organoclorados estão entre as poucas medidas práticas de prevenção primária que a mulher pode adotar para reduzir o risco de desenvolver câncer de mama.

Alguns estilos de vida têm sido sugeridos como associados a uma redução no risco de desenvolver câncer de mama: amamentar, ter um número elevado de partos, residir em área rural, praticar exercícios físicos e ingerir bebidas alcoólicas com moderação, manter uma alimentação equilibrada e controlar o peso. Tem-se especulado que a presença destes fatores leva a uma redução discreta no risco de desenvolver câncer de mama. Entretanto, devido à inconsistência dos resultados dos diferentes estudos epidemiológicos e à pequena magnitude das medidas de risco encontradas, ainda não podem ser associados, de forma inquestionável, à proteção. É importante ressaltar que vários destes fatores estão associados a um nível sócioeconômico baixo, o que pode contribuir para explicar menores taxas de incidência da doença em populações desfavorecidas.

\section{PreVEnção SECUNDÁRIA}

A prevenção secundária tem por finalidade alterar o curso da doença, uma vez que seu início biológico já aconteceu, por meio de intervençôes que permitam sua detecção precoce e seu tratamento oportuno. Para isso, deve haver clara evidência de que a doença em questão possa ser identificada em uma fase precoce, quando ainda não está clinicamente aparente, e que permita uma abordagem terapêutica eficaz, alterando seu curso ou minimizando os riscos associados com a terapêutica clínica. Além disso, a queda resultante em morbidade ou mortalidade deve ser alcançada sem um grande fardo de efeitos adversos gerados pela estratégia adotada. A detecção precoce de uma doença é possível por meio de educação para o diagnóstico precoce em pessoas sintomáticas ou do rastreamento (triagem) em populaçōes assintomáticas ${ }^{16}$.

É essencial educar a população e os profissionais de saúde para o reconhecimento dos sinais e sintomas precoces do câncer, contribuindo para sua detecção em estágios menos avançados e aumentando as chances de sucesso do tratamento preconizado. Isto pode ser obtido por meio de campanhas educativas e capacitação de agentes de saúde ${ }^{16}$.

Define-se rastreamento como o exame das pessoas assintomáticas objetivando identificar aquelas com maiores chances de apresentar uma doença ${ }^{17}$. Presumese a identificação de doença ou de alteração previamente desconhecida pelo paciente por meio da aplicação de testes que possam separar, dentre pessoas aparentemente saudáveis, aquelas que provavelmente têm a doença. Estas últimas serão encaminhadas para investigação diagnóstica. Assim, um teste de rastreamento não tem por fim fazer diagnóstico, mas indicar pessoas que, por apresentarem exames alterados ou suspeitos, devem ser encaminhadas para investigação diagnóstica. Alguns autores propõem que, para ser considerado como uma estratégia de rastreamento, é necessário que um teste não seja solicitado como resultado de uma queixa clínica específica do paciente, reforçando-se a necessidade de que as desordens sejam assintomáticas ${ }^{18}$. Por outro lado, o exame de pessoas com sintomas precoces inespecíficos para determinar se apresentam ou não doença pré-clínica ou em estágio inicial tem sido considerado por alguns autores como rastreamento.

Para que possa ser alvo de um programa de rastreamento, a doença deve constituir-se em um importante problema de Saúde Pública, apresentar uma fase pré-clínica detectável, mostrar benefícios quando o início do tratamento ocorre em sua fase pré-clínica em comparação ao seu início após os primeiros sintomas, ter um teste para rastreamento com níveis aceitáveis de acurácia, factibilidade, reproducibilidade e custo $^{19,20}$.

Quando o teste empregado por um programa de rastreamento detecta lesões precursoras, espera-se uma redução no número de casos novos de câncer (incidência) e conseqüentemente no número de óbitos (mortalidade), como o que se observa nos programas de rastreamento para o câncer do colo do útero ou do câncer de cólon e reto. Quando o objetivo é detectar a doença precocemente ou em sua fase pré-invasiva, estima-se que haja redução na mortalidade por câncer, como o que ocorre nos programas de rastreamento para o câncer de mama. Assim, em programas desenhados para detectar cânceres potencialmente invasivos em sua fase precoce, não haverá redução nas taxas de incidência. Ao contrário, a introdução das ações poderá seguir-se, inicialmente, de um aumento aparente nessas taxas, uma vez que casos prevalentes assintomáticos (isto é, já existentes no momento do exame) serão detectados. Somente após esse aumento inicial será observada uma queda nas taxas ${ }^{16}$.

Em geral, um impacto na taxa de incidência por câncer avançado poderá ser observado quatro anos após o início do programa de rastreamento, seguido por um impacto na taxa de mortalidade cerca de dois anos mais tarde. A redução da mortalidade por câncer de mama, conseqüente ao rastreamento mamográfico, geralmente é mais lenta em programas populacionais que nos ensaios clínicos. Isso se deve à exclusão das mortes por câncer de mama ocorridas em casos que haviam sido diagnosticados antes do convite para o rastreamento, à 
dificuldade em treinar todos os profissionais de saúde e obter sua adesão às práticas recomendadas e à demora para cobrir a população nacional ${ }^{16}$.

Os programas de rastreamento podem ser implantados de forma organizada ou oportunista. Conceitua-se como organizado o programa de rastreamento implementado por meio de um planejamento ativo, dispensado a pessoas convidadas, de grupos etários pré-definidos, com freqüência preestabelecida. Por outro lado, quando a ação de deteç̧ão precoce resulta de interação individual, por iniciativa pessoal ou do profissional de saúde, considerase como rastreamento oportunista ${ }^{16}$. Nesta última modalidade insere-se o achado de casos ("case finding"), que pode ocorrer por meio da utilização de um protocolo visando o diagnóstico precoce em pacientes que se apresentam para consulta clínica por razões não relacionadas à condição para a qual serão investigados. Estes podem ser pacientes saudáveis que comparecem para receber cuidados preventivos ou para o exame médico rotineiro ou ainda pacientes que se apresentam para diagnóstico e tratamento de outras condições sem conexão com a estratégia de detecção precoce utilizada. Alguns exemplos dessas estratégias são os programas multifásicos, nos quais são realizados diferentes testes mesmo que aparentemente não exista indicação clínica, os exames periódicos de saúde, que avaliam o estado de saúde e buscam sinais precoces de doença e os programas específicos, como a testagem da pressão arterial ou dos níveis de colesterol.

Além disso, o rastreamento é dito seletivo quando as açôes são dirigidas a uma pequena parcela da população, definida como de alto risco para uma doença, com o objetivo de reduzir substancialmente o número de resultados falso-positivos e os custos de sua aplicação ${ }^{21}$. Programas de rastreamento concentrados somente em pessoas identificadas como de alto risco raramente se justificam uma vez que os casos identificados nestas populações representam uma pequena proporção do total de casos existentes. Esta estratégia tem sido reconhecida como de baixa sensibilidade e de eficácia duvidosa ${ }^{16}$.

Os programas de ratreamento para câncer de mama têm como objetivo identificar mulheres que se encontram em estágio precoce da doença. Atualmente há três estratégias disponíveis para rastreamento do câncer de mama: mamografia (MMG), exame clínico das mamas (ECM) e auto-exame das mamas (AEM), que serão discutidas a seguir.

\section{Mamografia (MMG)}

O rastreamento do câncer de mama por meio de seu exame radiográfico tem sido usado com o intuito de identificar alteraçôes sugestivas de malignidade antes que ocorram manifestações clínicas. A MMG foi introduzida em larga escala populacional com fins de rastreamento organizado, inicialmente, na Islândia e em vários distritos da Suécia, em 1987. Em 1988 foi a vez dos Países Baixos e Canadá, enquanto a Finlândia iniciou seu programa em 1989. No início dos anos 90, programas organizados foram implantados na Austrália, Reino Unido, Estados Unidos, Israel e, posteriormente, França. Os benefícios em termos de redução da mortalidade por câncer de mama começam a ser notados cerca de quatro anos após o início do rastreamento e continuam a crescer por cerca de 10 anos, mantendo-se por todo o período de observação ${ }^{5}$.

Até este momento há nove ensaios clínicos com resultados publicados que avaliaram a eficácia da MMG em promover a redução da mortalidade por câncer de mama: "Health Insurance Plan Study" (HIP, Nova Iorque - EUA) ${ }^{22}$, Edimburgo (Escócia) ${ }^{23}$, "National Breast Screening Study"1 (NBSS1, Canadá) ${ }^{24,25}$, "National Breast Screening Study"2 (NBSS2, Canadá) ${ }^{25,25}$, Estocolmo (Suécia) $^{26}$, Gothenburgo (Suécia) ${ }^{27}$, "Malmo Mammography Screening Trial" (Suécia) ${ }^{28}$, Kopparburg e Ostergotland (dois distritos da Suécia) ${ }^{29}$. Estes estudos, iniciados entre 1963 e 1982, incluíram mulheres de 39 e 74 anos de idade, com intervalos entre exames que variaram de 12 a 34 meses. Na tabela 3 são apresentados os principais resultados dos estudos que avaliaram o impacto da MMG sobre a mortalidade por câncer de mama.

Nestes ensaios clínicos, a sensibilidade do exame mamográfico anual em detectar o câncer de mama variou entre 71 e $98 \%$, enquanto para o exame bienal esta variou entre 53 a $86 \%$, conforme a faixa etária; já a especificidade esteve entre 94 e $97 \%$ e o valor preditivo positivo variou de 2 a $22 \%$, tendo como referência a realização de um único exame.

Outros ensaios clínicos, com resultados ainda não publicados, estão sendo conduzidos no Reino Unido ${ }^{31}$, Singapura $^{32}$ e Eslovênia ${ }^{33}$.

Estes ensaios clínicos vêem apontando para os benefícios do rastreamento mamográfico de mulheres entre os 50 e os 69 anos de idade. Entretanto, as controvérsias têm se situado, sobretudo, na sua indicação a mulheres abaixo dos 50 anos de idade, por inconsistência dos resultados, e àquelas acima dos 70 anos de idade, por não estarem representadas na maioria dos estudos (apenas os estudos de Kopparburg e Ostergotland incluíram mulheres acima dos 65 anos de idade).

Em 2001, dois pesquisadores dinamarqueses, 
Tabela 3. Ensaios clínicos que avaliaram a mamografia como estratégia para o rastreamento do câncer de mama

\begin{tabular}{|c|c|c|c|c|c|c|c|c|}
\hline Estudo & $\begin{array}{l}\text { ano de } \\
\text { início }\end{array}$ & referência & $\begin{array}{l}\text { idade para } \\
\text { inclusão }\end{array}$ & $\begin{array}{l}\text { intervalo } \\
\text { entre } \\
\text { exames }\end{array}$ & $\begin{array}{l}\text { exame } \\
\text { clinico das } \\
\text { mamas }\end{array}$ & $\begin{array}{c}\text { tempo de } \\
\text { seguimento (até } \\
\text { 2002) }\end{array}$ & $\begin{array}{l}\text { risco relativo de } \\
\text { mortalidade por } \\
\text { câncer de mama }\end{array}$ & $\begin{array}{c}\mathrm{N}^{\circ} \text { de } \\
\text { mamografias } \\
\text { necessárias } \\
\text { para salvar uma } \\
\text { vida }\end{array}$ \\
\hline HIP & 1963 & $\begin{array}{l}\text { Shapiro et } \\
\text { al., } 1988^{22}\end{array}$ & $40-64$ & $12 m$ & Sim & 18 anos & 0,79 & 883 \\
\hline Edimburgo & 1978 & $\begin{array}{l}\text { Alexander et } \\
\text { al., } 1999^{23}\end{array}$ & $45-64$ & $24 m$ & Sim & 14 anos & $\begin{array}{c}0,79 \\
(0,60-1,02)\end{array}$ & 980 \\
\hline NBSS1 & 1980 & $\begin{array}{l}\text { Miller et al, } \\
1992^{24} \\
2002^{25}\end{array}$ & $40-49$ & $12 m$ & Sim & 13 anos & $\begin{array}{c}0,97 \\
(0,74-1,27)\end{array}$ & - \\
\hline NBSS2 & 1980 & $\begin{array}{l}\text { Miller et al, } \\
1992^{24} \\
2002^{25}\end{array}$ & $50-59$ & $12 m$ & Sim & 13 anos & $\begin{array}{c}1,02 \\
(0,78-1,33)\end{array}$ & - \\
\hline Estocolmo & 1981 & $\begin{array}{c}\text { Frisell et al., } \\
1997^{26}\end{array}$ & $40-64$ & $28 m$ & Não & 14 anos & $\begin{array}{c}0,91 \\
(0,65-1,27)\end{array}$ & 3.468 \\
\hline Gothenburgo & 1982 & $\begin{array}{l}\text { Bjurstam et } \\
\text { al., } 1997^{27}\end{array}$ & $39-59$ & $18 m$ & Não & 12 anos & $\begin{array}{c}0,76 \\
(0,56-1,04)\end{array}$ & 1.139 \\
\hline Malmo & $1976-78$ & $\begin{array}{c}\text { Anderson \& } \\
\text { Janzon, } \\
1997^{28}\end{array}$ & $40-69$ & $18-24 m$ & Não & $11-13$ anos & $\begin{array}{c}0,82 \\
(0,67-1,00)\end{array}$ & 584 \\
\hline Kopparburg & 1977 & $\begin{array}{c}\text { Tabar et al, } \\
1995^{29}\end{array}$ & $40-74$ & $24-33 m$ & Não & \multirow{2}{*}{20 anos } & \multirow{2}{*}{$\begin{array}{c}0,68 \\
(0,59-0,80)\end{array}$} & \multirow{2}{*}{553} \\
\hline Ostergotland & 1978 & $\begin{array}{c}\text { Tabar et al, } \\
1995^{29}\end{array}$ & $40-74$ & $24-34 m$ & Não & & & \\
\hline
\end{tabular}

Adaptado de : Humphrey et al, 200230e IARC, 200235.

OLSEN \& GOTZSCHE ${ }^{34}$, em uma revisão sistemática da literatura realizada para a Cochrane Library, concluíram pela ausência de eficácia do rastreamento mamográfico, após a exclusão de estudos considerados enviesados e que, conseqüentemente, comprometiam os resultados. Nesta revisão somente foram incluídos o "Canadian National Breast Screening Study" e o "Malmo Mammography Screening Trial". Com isso, o efeito estimado da MMG sobre todas as causas de morte foi zero. Além disso, estes autores apontaram para uma incidência de mastectomias e tumorectomias 30 a $40 \%$ maior no grupo submetido ao rastreamento mamográfico, o que representa 40 cirurgias adicionais para cada 10.000 mulheres rastreadas.

Posteriormente, em reunião promovida pela Organização Mundial de Saúde ${ }^{35}$, em Lyon - França, de 5 a 12 de março de 2002, o grupo de trabalho de avaliação das estratégias de prevenção do câncer concluiu que o exame radiográfico das mamas de mulheres entre 50 e 69 anos, quando realizado a intervalos de um a dois anos (considerou-se que o intervalo não altera o efeito protetor) promove uma redução de $25 \%$ (risco relativo de morte de 0,75 , com intervalo com $95 \%$ de confiança $=0,67-0,85)$ nas taxas de mortalidade por este câncer. Para mulheres com idade entre 40 e 49 anos a síntese dos resultados dos ensaios clínicos mostrou uma possível ausência de efeito; o risco relativo de morte obtido dos estudos que incluíram este grupo etário e usaram MMG isoladamente foi de 0,81 (intervalo com $95 \%$ de confiança $=0,65-1,01)$. Ambas reduçōes foram estatisticamente significativas. Nesta revisão foram incluídos, além dos ensaios clínicos em que a MMG foi usada isoladamente, os resultados do programa finlandês de prevenção do câncer de $\mathrm{mama}^{36}$.

Em setembro 2002, com a atualização das observações dos ensaios clínicos em andamento ${ }^{25,37}$, o grupo de estudos de câncer de mama da força tarefa norte-americana ${ }^{30}$ ratificou evidências em favor da redução da mortalidade por câncer de mama em mulheres submetidas a MMG anual ou bienal, associada ou não ao ECM. Para o grupo de mulheres de 50 a 69 anos estimou-se uma redução da mortalidade de $16 \%$ (Risco relativo = 0,84; intervalo com $95 \%$ de confiança $=$ $0,77-0,91)$, enquanto para mulheres na faixa etária compreendida entre 40 e 49 anos de idade, a redução esperada é de $15 \%$ (Risco relativo= 0,85 ; intervalo com $95 \%$ de confiança $=0,73-0,99)$.

Não está claro porque o efeito do rastreamento aparece mais lentamente em mulheres mais jovens. Especula-se que seja devido ao fato de que as mulheres incluídas nos ensaios clínicos com menos de 50 anos de idade continuam a se submeter ao exame mamográfico após completarem esta idade e que há fenômenos biológicos que só vão ocorrer por volta dos 50 anos de idade, como a menopausa. Além disso, em mulheres nas faixas etárias mais jovens observa-se uma menor incidência do câncer de mama, um crescimento mais rápido dos tumores e uma sensibilidade inferior do exame mamográfico 5 .

Ainda segundo o grupo de estudos de câncer de mama da força tarefa norte-americana ${ }^{30}$, para salvar uma vida seria necessário examinar 1.224 e 1.792 mulheres, 
conforme se encontrem na faixa etária que vai dos 40 aos 49 ou 50 a 69 anos, respectivamente. Estes autores concluíram que, para mulheres acima de 70 anos de idade, não há informação suficiente para saber se o rastreamento mamográfico tem algum impacto sobre as taxas de mortalidade. Apesar disso, a força tarefa norte-americana recomenda que mulheres acima dos 70 anos de idade submetam-se a MMG anual ou bienal, a menos que alguma doença grave reduza sua expectativa de vida.

Outras tecnologias de imagem (por exemplo: ultrasom, MMG digital, cintigrafia e ressonância magnética) estão sendo investigadas, não havendo, neste momento, evidências científicas que comprovem sua eficácia como método para rastreamento populacional do câncer de mama 5 .

\section{Exame Clínico das Mamas (ECM)}

Até o presente momento não há evidências científicas da eficácia do ECM na redução da mortalidade por câncer de mama. Dos nove ensaios clínicos realizados para avaliar a eficácia do rastreamento mamográfico, quatro incluiram o ECM como intervenção complementar. Entretanto, somente o NBSS2 comparou as duas manobras em mulheres entre 50 e 59 anos de idade $^{24}$. Das 39.405 mulheres incluídas no estudo, metade recebeu exame mamográfico acrescido de ECM enquanto as demais somente o ECM. Após 13 anos de seguimento, não houve diferença significativa no número de casos diagnosticados e na evolução para óbito (107 versus 105), sugerindo que o ECM é tão efetivo quanto a $\mathrm{MMG}^{39}$.

No Japão, o rastreamento do câncer de mama por meio do exame clínico das mamas, iniciado no final dos anos 80 , mostrou que em comunidades em que a cobertura do exame atingiu cerca de $50 \%$ das mulheres adultas (30 a 69 anos de idade), houve uma redução de 42\% na mortalidade por câncer de mama, comparandose os períodos 1986-1990 e 1991-199540.

Já na Alemanha, o "Munich Study", realizado entre 1996 e 1998, mostrou que a redução da mortalidade por câncer de mama estimada em 10 anos foi de 25,1\% quando somente o exame clínico foi utilizado para rastreio contra $44,8 \%$ quando foi utilizada a mamografia ${ }^{41}$.

Uma recente revisão bibliográfica ${ }^{42}$ mostrou, com base nos dados dos ensaios clínicos para avaliar a eficácia do rastreamento mamográfico para o câncer de mama, que a sensibilidade do ECM variou entre 40 e $69 \%$, a especificidade esteve entre 88 e $99 \%$, enquanto o valor preditivo positivo do exame ficou entre 4 e $50 \%$. Os mais altos valores de sensibilidade são obtidos quando o exame é realizado durante pelo menos 5 a 10 minutos ${ }^{43}$.

Em suas recomendações, a Sociedade Americana de Câncer propõe que o ECM seja iniciado aos 20 anos de idade, com uma periodicidade trienal até os 39 anos, quando então deve passar a ser realizado anualmente ${ }^{19,44}$.

Uma das limitaçôes do uso do ECM é que tumores em estágio I, com menos de $2 \mathrm{~cm}$ de diâmetro, podem ser impalpáveis. Por outro lado, a análise dos resultados do programa norte-americano de detecção precoce do câncer do colo do útero e mama, para os anos 19951998, mostrou que 11,2\% dos cânceres de mama não apresentaram imagem radiológica perceptível, sendo detectados exclusivamente pelo $\mathrm{ECM}^{45}$.

\section{Auto Exame das Mamas (AEM)}

$\mathrm{O}$ auto exame sistemático das mamas tem sido recomendado desde a década de $30^{46} \mathrm{e}$ foi incorporado nas políticas de saúde pública norte-americanas desde os anos $50^{47}$. Considerando-se que até $90 \%$ dos casos de câncer de mama são detectados pelas próprias mulheres ${ }^{10}$, pode-se deduzir que a promoção do AEM seja uma estratégia eficaz para sua detecção.

Embora já tenham sido realizados mais de 30 estudos não randomizados sobre o uso do AEM para o rastreamento do câncer de mama, não há evidências científicas incontestáveis de que sua prática promova a redução da mortalidade por este câncer. Diversos estudos de tipo caso-controle apontaram para uma leve redução na mortalidade em pacientes submetidas ao AEM, enquanto estudos de coorte e ensaios clínicos não suportaram esses achados. Em um ensaio clínico não randomizado, iniciado em 1973 na Inglaterra pelo "UK Early Detection of Breast Cancer Study Group", não foi constatada redução nas taxas de mortalidade por este câncer ${ }^{48}$. Há apenas dois ensaios clínicos randomizados que avaliaram o impacto do AEM sobre a mortalidade por câncer de mama. O primeiro foi iniciado em 1985, em Moscou e São Petersburgo ${ }^{49,50}$, enquanto o segundo teve início em 1989, em Xangai51. Ambos envolveram um grande número de mulheres que foram meticulosamente treinadas e receberam reforços para garantir o aprendizado da técnica. Após um seguimento de 5 a 10 anos, ambos apontaram para a ineficácia do AEM em reduzir a mortalidade pelo câncer de mama. Em outubro de 2002, a publicação dos resultados finais deste último ensaio clínico confirmou as observaçôes anteriores, concluindo-se pela incapacidade do AEM em alterar as taxas de mortalidade pelo câncer de mama. Uma síntese destes estudos é apresentada na tabela 4 . 
Tabela 4. Síntese dos estudos experimentais sobre a eficácia do AEM na redução da mortalidade por câncer de mama.

\begin{tabular}{|l|c|c|c|c|c|c|}
\hline $\begin{array}{c}\text { Local do } \\
\text { estudo }\end{array}$ & $\begin{array}{c}\text { Ano do } \\
\text { Início }\end{array}$ & $\begin{array}{c}\text { Faixa } \\
\text { etária }\end{array}$ & $\begin{array}{c}\text { Número de } \\
\text { mulheres }\end{array}$ & $\begin{array}{c}\% \text { de } \\
\text { participação }\end{array}$ & $\begin{array}{c}\text { Tempo de } \\
\text { Seguimento }\end{array}$ & $\begin{array}{c}\text { RR de morte por câncer } \\
\text { de mama (IC 95\%) }\end{array}$ \\
\hline Inglaterra $^{48}$ & 1979 & $45-64$ anos & 253.219 & $30-53 \%$ & 9 a 9,8 anos & $1,07(0,93-1,22)$ \\
\hline Xangai $^{51}$ & 1989 & $31-64$ anos & 266.064 & $98 \%$ & 5 anos & $1,04(0,82-1,33)$ \\
\hline Rússia $^{50}$ & 1985 & $40-64$ anos & 120.310 & $76,4 \%$ & 10 anos & $0,95(0,76-1,19)$ \\
\hline
\end{tabular}

Embora a sensibilidade do AEM nunca tenha sido formalmente avaliada, estimativas indicam que de cada 100 casos de câncer de mama, o AEM detecta 26, o ECM detecta 45 e a MMG $71^{47}$.

Apesar de não haver evidência direta de que o AEM efetivamente reduza a mortalidade por câncer de mama, é importante que as mulheres mantenham-se atentas às alterações em suas mamas e reportem qualquer alteração ao profissional de saúde ${ }^{46}$, reconhecendo-se, desta forma, a efetividade potencial do estímulo à sua prática como estratégia complementar ${ }^{10}$. Embora as mulheres, individualmente, possam ver a realização do AEM como parte de seu auto-cuidado ${ }^{52}$, as recomendações para profissionais de saúde devem enfatizar que não há evidências científicas de que o AEM reduza a mortalidade por câncer de mama e que do ponto de vista ético deve-se otimizar a utilização dos recursos públicos em açõos coletivas de eficácia comprovada ${ }^{52,53}$.

\section{CONSIDERAÇÕES FINAIS}

Os dados reunidos neste trabalho visam contribuir para um melhor conhecimento da efetividade das açôes de prevenção do câncer de mama feminino. É importante ressaltar que não houve intenção de esgotar o assunto e que a revisão da literatura não foi realizada de forma sistemática o que, conseqüentemente, implicou a ausência de estratégias de controle da qualidade e de definição de critérios para a seleção dos estudos citados.

Com base nas informações disponíveis, fica evidente que os principais fatores de risco para o câncer de mama na mulher (idade, história familiar, história patológica pregressa, história reprodutiva, suscetibilidade genética etc) não são passíveis de intervenção em nível populacional. Entretanto, um pequeno número de fatores de risco poderá ser alvo das estratégias de prevenção primária para o câncer de mama, entre eles obesidade, tabagismo, ooforectomia e exposição à radiação iônica e aos pesticidas/organoclorados. Cumpre ressaltar que, à exceção da ooforectomia, todos os demais têm mostrado uma associação frágil com o câncer de mama, sendo capazes de explicar um pequeno percentual de casos. Logo, entre as possíveis recomendaçôes para a população em geral está o estímulo a hábitos de vida saudáveis, o que inclui não fumar, manter uma alimentação equilibrada, ingerir bebidas alcoólicas com moderação e manter o peso ideal. Acrescente-se a isso cuidados nos ambientes de trabalho sobretudo no que diz respeito à exposição à radiação iônica e aos pesticidas.

No que diz respeito ao rastreamento populacional para o câncer de mama, o presente nível de evidência científica permite concluir que a recomendação de MMG acompanhada do ECM, a cada um ou dois anos, nas mulheres entre 50 e 69 anos de idade, tem se mostrado a estratégia mais eficaz na redução da mortalidade por câncer de mama. Há que se observar que o rastreamento do câncer de mama pela MMG é intrinsecamente menos efetivo que o rastreamento do câncer do colo do útero por meio do exame citopatológico. Estimam-se, para essas estratégias, reduções da mortalidade da ordem de 25 e $70 \%$, respectivamente. Além disso, a Organização Mundial da Saúde ${ }^{16}$ recomenda que o rastreamento para o câncer de mama deva ser iniciado somente quando houver disponibilidade de acesso à MMG para pelo menos 70\% da população alvo e reconhece que, frente aos atuais níveis de evidência, um programa nacional de controle do câncer de mama não deve recomendar o rastreamento por AEM e ECM. Estima-se que a efetividade do ECM seja menor que aquela da MMG, considerando-se que sua sensibilidade tem variado entre 40 e 69\%. Por ser uma técnica de difícil padronização, observa-se variação da reprodutibilidade, na dependência do profissional que a executa.

Entretanto, a Organização Mundial da Saúde ${ }^{16}$ recomenda que, em países onde o rastreamento mamográfico não está disponível, deve-se encorajar o diagnóstico precoce do câncer de mama por meio do ECM, com ênfase em mulheres que frequentam serviços de saúde por outras razôes, especialmente quando estiverem na faixa etária compreendida entre os 40 e os 69 anos de idade (rastreamento oportunístico). 


\section{AGRADECIMENTOS}

A Moyses Szklo (Johns Hopkins University - EUA) e Zulmira Hartz (FIOCRUZ - Rio de Janeiro) pelas valiosas críticas e sugestóes apresentadas ao texto original.

\section{REFERÊNCIAS BIBLIOGRÁFICAS}

1. Level HR, Clark EG. Medicina preventiva. McGraw-Hill do Brasil: São Paulo; 1976.

2. Grundy PF. A rational approach to the "at risk" concept. Lancet 1973;2(7844):1489.

3. Taubes G. Epidemiology faces its limits. Science 1995;269:164-9.

4. Meister K, Morgan J. Risk factors for breast cancer: a report by the American Council on Science and Health. 2000.

5. Fletcher SW. Screening for breast cancer. Up to date. 2003. Available from: URL: http://www.uptodate.com

6. Minister of Public Works and Government Services (Canada). Review of lifestyle and environmental risk factors for breast cancer: summary report. 2001

7. Associação de Combate ao Câncer em Goiás (Brasil). Registro de câncer de base populacional de Goiânia. Câncer em Goiânia: tendências (1988 - 1997). Goiânia; 2000.

8. Fundação Oncocentro de São Paulo (Brasil). Incidência de câncer no município de São Paulo, Brasil: 1997-1998. Mortalidade de câncer no Município de São Paulo, Brasil: tendência no período 1969-1998. São Paulo; 2001.

9. Fundação Centro de Controle de Oncologia do Amazonas (Brasil). Registro de câncer de base populacional de Manaus: dados de 1999. Manaus: FCECON; 2002.

10. Robles SC, Galanis E. Breast cancer in Latin America and the Caribbean. PAHO/WHO; 2001.

11. McPherson K, Steel CM, Dixon JM. ABC of breast diseases: breast cancer-epidemiology, risk factors, and genetics. BMJ 2000;321:624-8.

12. Fletcher SW. Risk factors for breast cancer. Up to date. 2002. Available from: URL: http://www.uptodate.com

13. Fundação Nacional de Saúde. Centro Nacional de Epidemiologia (Brasil). Sistema de informação sobre mortalidade 1979 - 1997: dados de declaração de óbito. Disponível em: URL:www.datasus.gov.br

14. Thuler LCS , Leal PR, Costa CR. Influência do silicone na gênese do câncer de mama. Rev Bras Cancerol 2003;49(1):9-15.

15. Madigan MP, Ziegler RG, Benichou J, Byrne C, Hoover RN. Proportion of breast cancer cases in the United States explained by well-established risk factors. J Natl Cancer Inst 1995;87(22):1681-5.

16. World Health Organization. National Cancer Control Programmes: polices and managerial guidelines. $2 \mathrm{nd}$ ed. Geneva: WHO; 2002.
17. Rothman KJ, Greenland S, editors. Modern epidemiology. Philadelphia: Lippincott-Raven; 1998.

18. 18. Department of public health sciences (UK). Guy's King's and St Thomas' Medical School. Screening. 2003. Available from: URL:http://www-phm.umds.ac.uk/teaching/ClinEpid/Screen.htm

19. Eyre HJ, Smith RA, Mettlin CJ. Cancer screening and early detection. In: Decker BC. Cancer medicine. 5th ed. London; 2000.

20. Blamey RW, Wilson RM, Patnick J. ABC of breast diseases. Screening for breast cancer. BMJ 2000;321:689-93.

21. Szklo M. Selective screening: when should screening be limited to high-risk individuals? J Gen Intern Med 1990;5:S47-9.

22. Shapiro S, Venet W, Strax P, Venet L. Current results of the breast cancer screening randomized trial: the health insurance plan (HIP) of greater New York study. In: Day NE, Miller AB, editors. Screening for breast cancer. Toronto: Hans Huber; 1988. p. 3-15.

23. Alexander FE, Anderson TJ, Brown HK, Forrest AP, Hepburn W, Kirkpatrick AE, et al. 14 years of follow-up from the Edinburgh randomised trial of breast-cancer screening. Lancer 1999;353:1903-8.

24. Miller AB, Baines CJ, To T, Wall C, Canandian National Breast Screening Study: 1- Breast cancer detection and death rates among women aged 40 to 49 years and 50 to 59 years. CMAJ 1992;147:1459-88.

25. Miller AB, To T, Baines CJ, Wall C. Canadian National Breast Screening Study 1: breast cancer mortality after 11 to 16 years of follow-up. A randomized screening trial of mammography in women age 40 to 49 years. Ann Intern Med 2002;137:305-12.

26. Frisell J, Lidbrink E, Hellströn L, Rutqvist LE. Follow after 11 years-update of mortality results in the Stockholm mammographic screening trial. Breast Cancer Res Treat 1997;45:263-70.

27. Bjurstan N, Björneld L, Duffy SW, Smith TC, Cahlin E, Erikson O, et al. The Gothenburg Breast Cancer Screening Trial: preliminary results on breast cancer mortality for women aged 39-49. Monogr Natl Cancer Inst 1997:53-5.

28. Anderson I, Janzon L. Reduced breast cancer mortality in women under age 50: update results from the Malmö Mammographic Screening Program. Monogr Natl Cancer Inst 1997:63-7.

29. Tabar L, Fagerberg G, Chen HH, Duffy SW, Smart CR, Gad A, et al.Efficacy of breast cancer screening by age. New results from the Swedish Two Country Trial. Cancer 1995;75:2507-17.

30. Humphrey LL, Helfand M, Chan BKS, Woolf SH. Breast cancer screening: a summary of the evidence for the U.S. preventive services task force. Ann Intern Med 2002;137(5):347-62.

31. Moss S. A trial to study the effect on breast cancer mortality 
of annual mammographic screening in women starting at age 40. J Med Screen 1999;6:144-8.

32. Ng EH, Ng Fc, Tan PH, Low SC, Chiang G, Tan KP, et al. Results of intermediate measures from a population-based, randomized trial of mammographic screening prevalence and detection of breast carcinoma among Asian women : The Singapore Breast Screening Project. Cancer 1998;82:1521-8.

33. Rudolf Z. Pilot study of breast cancer screening in six communes of Slovenia. In: Sankaranarayanan R, Wahrendorf J, Demaret $E$, editors. Diretory of ongoing research in cancer epidemiology. Lyon: International Agency for Research on Cancer; 1994. (IARC Scientific Publications; no. 130).

34. Olsen O, Gotzsche PC. Cochrane review on screening for breast cancer with mammography [letter]. Lancet 2001;358:1340-2.

35. International Agency for Research on Cancer. Breast cancer screening. Lyon: IARC; 2002. (IARC Handbooks of Cancer Prevention; vol. 7).

36. Hakama M, Pukkala E, Heikkila M, Kallio M. Effectiveness of the public health policy for breast cancer screening in Finland: population based cohort study. BMJ 1997;314:864-7.

37. Nyström L, Andersson I, Bjurstam N, Frisell J, Nordenskjöld $B$, Rutqvist LE. Long-term effects of mammography screening: update overview of the Swedish randomised trials. Lancet 2002;359:909-19.

38. Ministério da Saúde. Instituto Nacional de Câncer (Brasil). Estimativas da incidência e mortalidade por câncer no Brasil, 2003. Rio de Janeiro: INCA; 2003.

39. Miller AB, To T, Baines CJ, Wall C. Canadian National Breast Screening Study-2: 13-year results of a randomized trial in women aged 50-59 years. J Natl Cancer Inst 2000;92(18):1490-9.

40. Kuroishi T, Hirose K, Suzuki T, Tominaga S. Effectiveness of mass screening for breast cancer in Japan. Breast Cancer 2000;7:1-8.

41. Engel J, Baumert J, Dirschedl P, Sauer H, Hölzel D. Effectiveness of self-examination, palpation and mammography for the early detection of breast cancer: first results of the Munich Field Study. Geburtshilfe Frauenheilkd 2000; 60:155-64.

42. Barton MB, Harris R, Fletcher SW. The rational clinical examination. Does this patient have breast cancer? The screening clinical breast examination: should it be done? How? JAMA 1999; 282:1270-80.

43. Huffman GB. Should family physicians do screening breast exams? American Family Physician; 2000. Available from: URL: www.aafp.org

44. American Cancer Society. Cancer prevention \& early detection. Facts Figures [serial on line] 2003. Available from: URL: http://www3.cancer.org/

45. Bobo JK, Lee NC, Thames SF. Findings from 752,081 clinical breast examinations reported to a National Screening Program from 1995 through 1998. J Natl Cancer Inst 2000;92(12):971-6.

46. Austoker J. Breast self examination does not prevent deaths due to breast cancer, but breast awareness is still important. BMJ 2003;326:1-2.

47. Fletcher SW, Black W, Harris R, Rimer BK, Shapiro S. Report of the international workshop on screening for breast cancer. J Natl Cancer Inst 1993;85(20):1644-56.

48. Ellman R, Moss SM, Coleman D, Chamberlain J. Breast self-examination programmes in the trial of early detection of breast cancer: ten years findings. Br J Cancer 1993;68:208-12.

49. Semiglazov VF, Sagaidak VN, Moiseenko VM, Mikhailov EA. Study of the role of breast self-examination in the reduction of mortality from breast cancer: The Russian Federation/World Health Organization Study. Eur J Cancer 1992;29A:2039-46.

50. Semiglazov VF, Moiseenko VM, Manikhas AG, Protsenko SA, Kharikova RS, Seleznev IK, et al. A prospective randomized trial (St-Petersburg, WHO) of the role of selfexamination in early detection of breast cancer. Rus J Oncol 2000; (2):4-9.

51. Thomas DB, Gao DL, SelfSG, Allison CJ, Tao Y, Mahloch $\mathrm{J}$, et al. Randomized trial of breast self-examination in Shanghai: methodology and preliminary results. J Natl Cancer Inst 1997;89(5):355-65.

52. Clarke V, White V, Savage S, Hill D, Jolley D. The effectiveness of breast self-examination: a literature review. $\mathrm{Na}$ tional Breast Cancer Center (Australia). 1999. Available from: URL: http://www.nbcc.org.au/

53. Schabas R. Is public health ethical? Rev Can Santé Publique 2002;93(2):98-100. 